\title{
CONTROVERSIAL ISSUES IN THE TREATMENT OF DYSLIPIDEMIAS IN PATIENTS WITH DIABETES MELLITUS
}

\author{
Klaus G. Parhofer \\ Medizinische Klinik IV - Grosshadern, University of Munich, Munich, Germany
}

\begin{abstract}
Patients with diabetes mellitus have an elevated cardiovascular risk. Lipid-lowering therapy is a successful strategy to prevent atherosclerotic events in these patients. Therefore, almost all professional societies recommend statin therapy for patients with diabetes under certain conditions. Despite this broad consensus, a number of controversial issues remain. Thus, it remains unclear in which patients the lipid parameters should be determined in the fasting state and in which postprandial values are sufficient. It is also an open issue whether all patients with diabetes should receive statin therapy and which goals should be achieved. While the benefit of statin-ezetimibe and statin-PCSK9-inhibition combinations has been shown in large outcome trials, results of outcome trials involving statins with triglyceride lowering drugs have been ambiguous. Thus, it is currently unclear which patients benefit from such combinations. Finally, the best strategy to address severe hypertriglyceridemia in patients with diabetes is unclear. This article discusses these issues and aims to provide help and information to practicing physicians taking care of patients with diabetes mellitus. (REV INVEST CLIN. 2018;70:237-43)
\end{abstract}

Key words: Diabetic dyslipidemia. Postprandial. Non-high-density lipoprotein -cholesterol.

\section{INTRODUCTION}

Treatment of dyslipidemia (mostly with statins) is a cornerstone of atherosclerosis prevention. Numerous studies have shown that cardiovascular morbidity and mortality can be reduced if the lipid profile is improved $^{1}$. Inadequate settings, it was even shown that total mortality can be decreased. The beneficial effect of lipid modification on cardiovascular morbidity and mortality is detectable in almost all subgroups

\section{Corresponding author:}

*Klaus G. Parhofer

Medizinische Klinik IV - Grosshadern

University Munich

Marchioninistraße 15, 81377 Munich

Germany

E-mail: klaus.parhofer@med.uni-muenchen.de but is particularly relevant in patient groups with high or very high risk. In that context patients with diabetes are a prime target for lipid-modifying therapy, and it is little surprising that all professional diabetes societies recommend, in one-way or another, lipid-lowering therapy in patients with diabetes mellitus ${ }^{2,3}$. Since the evidence is best for statin-based low-density lipoprotein (LDL)-cholesterol lowering this is the focus of most recommendations.

Received for publication: 02-05-2018 Approved for publication: 13-06-2018 doi: $10.24875 / R I C .18002574$ 
Table 1. Fasting versus non-fasting assessment of lipid profile according to EAS recommendations ${ }^{8}$

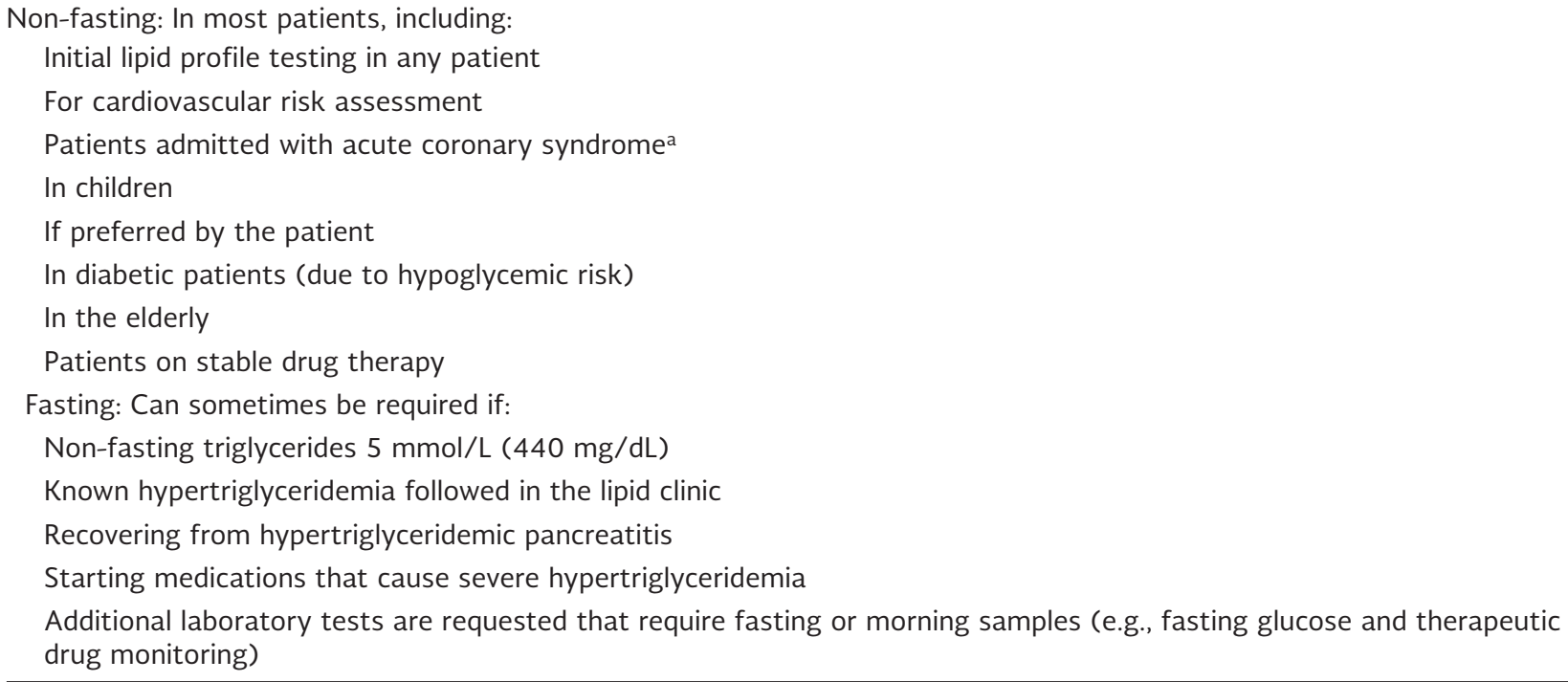

aWill need repeated lipid profile testing later because of acute coronary syndrome.

Despite this very convincing evidence, a number of controversial issues remain, which will be discussed in the subsequent paragraphs.

\section{FASTING OR NON-FASTING LIPID PROFILE}

In the past, there was a clear recommendation that lipid values should be determined in a fasting state (10-12 h postprandially). However, for a number of reasons, this dogma has been challenged. Most humans are in a postprandial state most of the time on most of the days. Even in normolipidemic subjects, it can be assumed that postprandial lipoproteins are present in plasma up to $10 \mathrm{~h}$ after the last fat-containing meal ${ }^{4}$. Therefore, most people will only be in a true fasting state very few hours per day. At the same time, it was shown that postprandial lipoproteins are pro-atherogenic and that this atherogenic potential can be estimated from the apoB or nonhigh-density lipoprotein ( $\mathrm{HDL}$ )-cholesterol concentration $^{5}$. Thus, from the point of view of prevention, it would be preferable to estimate risk in the postprandial state.

However, there is additionally a downside to measuring the lipid profile in a non-fasting state, particularly in patients with hypertriglyceridemia; triglyceride and thus also non-HDL-cholesterol varies considerably, depending on the content and the time of the last meal. Therefore, if the lipid profile of a hypertriglyceridemic patient is tested postprandially and has varied compared to the previous determination, it may be difficult to identify whether there is "real change" or whether the observed changes reflect other factors (such as less fat in the preceding meal).

The reason why fasting values were the gold standard in the past not only relates to the fact that triglyceride levels can be better compared (standardized situation) but also more importantly to the use of the Friedewald et al. formula to calculate LDLcholesterol $^{6}$. When the Friedewald et al. formula is used, it is absolutely necessary that patients are in a fasting state; otherwise, LDL-cholesterol will be underestimated. Now, with the widespread availability of direct LDL-cholesterol measurements, this is rarely a limitation.

Taken together, these findings have resulted in a position paper of the EAS recommending in which patients non-fasting values can be determined (Table 1$)^{7,8}$. This also applies to diabetic patients. However, since many diabetic patients come to the office and also have their fasting glucose level determined we usually recommend fasting lipids in our diabetic patients. 
Table 2. Recommendations by different professional societies on lipid-lowering treatment in patients with diabetes Recommendations by American Diabetes Association²

\begin{tabular}{|c|c|c|c|}
\hline Age & ASCVDa & Recommendations & $\gtrsim$ \\
\hline \multirow[t]{2}{*}{$<40$ year } & No & \multicolumn{2}{|c|}{ No statin therapy but can be considered if additional risk factors are present } \\
\hline & Yes & High-dose statin therapy ${ }^{\mathrm{b}}$ & () \\
\hline \multirow[t]{2}{*}{$\geq 40$ year } & No & Moderate intensive statin therapy but high-dose & \\
\hline & Yes & High-dose statin therapy ${ }^{b}$ & $\frac{1}{a}$ \\
\hline
\end{tabular}

aSCVD: Atherosclerotic cardiovascular disease.

bIf LDL-cholesterol $\geq 70 \mathrm{mg} / \mathrm{dL}(1.8 \mathrm{mmol} / \mathrm{L})$ despite maximally tolerated statin dose additional LDL-lowering therapy (such as ezetimibe or PCSK9-inhibition) should be considered.

Recommendations by the European Society of Cardiology (ESC) and the European Atherosclerosis Society (EAS) ${ }^{3}$

\begin{tabular}{|c|c|c|}
\hline Risk & Definition & Goal category \\
\hline Very high risk & $\begin{array}{l}\text { Diabetic patients with - ASCVD }{ }^{\mathrm{a}} \text { or } \\
\text { additional risk factors }{ }^{\mathrm{b}} \text { or endorgan } \\
\text { damage }^{\mathrm{c}}\end{array}$ & $\begin{array}{l}<70 \mathrm{mg} / \mathrm{dL}(<1.8 \mathrm{mmol} / \mathrm{L}) \text { or } \mathrm{LDL} \text { reduction } \\
\text { by } \geq 50 \% \text { if baseline } \mathrm{LDL} \text {-cholesterol is between } \\
70 \text { and } 135 \mathrm{mg} / \mathrm{dL}(1.8 \text { and } 3.5 \mathrm{mmol} / \mathrm{L}\end{array}$ \\
\hline High risk & $\begin{array}{l}\text { Diabetic patients without ASCVD, additional } \\
\text { risk factors or end organ damage }\end{array}$ & $\begin{array}{l}<100 \mathrm{mg} / \mathrm{dL}(<2.6 \mathrm{mmol} / \mathrm{L}) \text { or } \mathrm{LDL} \text { reduction } \\
\text { by } \geq 50 \% \text { if baseline } \mathrm{LDL}-\mathrm{cholesterol} \text { is between } \\
100-200 \mathrm{mg} / \mathrm{dL}(2.6 \text { and } 5.2 \mathrm{mmol} / \mathrm{L})\end{array}$ \\
\hline
\end{tabular}

${ }^{a}$ ASCVD: atherosclerotic, cardiovascular disease.

bRisk factors: smoking, hypertension or dyslipidemia.

'End organ damage: proteinuria.

\section{LIPID GOALS IN PATIENTS WITH DIABETES}

While the overall goal of lipid-lowering therapy (cardiovascular prevention) is very obvious, different approaches have been proposed to achieve this aim. From a practical point of view, there is little difference between the guidelines as in both cases most if not all diabetic patients should be treated with statins (Table 2).

The American Diabetes Association recommends high-dose statin therapy in all diabetic patients who either have established atherosclerotic disease and/or have an LDL-cholesterol concentration $\geq 190 \mathrm{mg} / \mathrm{dL}$ ( $\geq 4.9 \mathrm{mmol} / \mathrm{L}$ ) and/or are between 40 and 75 years of age ${ }^{2}$. Similarly, they also recommend statins in younger and older patients with diabetes, but state that the evidence from intervention trials is less solid in these age groups. In the newest recommendation, it is also stated that non-statin therapy such as ezetimibe and PCSK9 inhibitors should be considered if $\mathrm{LDL}$-cholesterol is $\geq 70 \mathrm{mg} / \mathrm{dL}(1.8 \mathrm{mmol} / \mathrm{L})$ on statin therapy.
The European professional societies (ESC/EAS) define specific lipid goals and simultaneously recommend an LDL-cholesterol reduction of at least $50 \%^{3}$ ESC/EAS differentiates between diabetic patients at very high risk and those at high risk. Patients at very high risk are those with established atherosclerotic disease and/or end organ damage, such as nephropathy, and/ or additional risk factors for whom an LDL-cholesterol $<70 \mathrm{mg} / \mathrm{dL}(1.8 \mathrm{mmol} / \mathrm{L})$ is recommended. For patients at high risk (diabetic patients without the above-mentioned characteristics), the LDL-cholesterol goal is $<100 \mathrm{mg} / \mathrm{dL}(2.6 \mathrm{mmol} / \mathrm{L})$. In both groups, a $>50 \%$ reduction from baseline LDL-cholesterol level is recommended.

While the focus is still very much on LDL-cholesterol levels, apoB and non-HDL-cholesterol levels have been defined as secondary goals. This reflects the fact that not only LDL but also all apoB containing lipoproteins (if they are not too large) are pro-atherogenic. This includes VLDL, IDL, and chylomicron-remnants. A number of studies have, therefore, shown that apoB and non-HDL-cholesterol levels better predict atherosclerotic disease than LDL-cholesterol levels ${ }^{9}$. While 
in patients with normotriglyceridemia apoB and LDLcholesterol strongly correlate to each other, fewer correlations are observed in patients with combined or diabetic dyslipidemia (elevated LDL-cholesterol and elevated triglycerides). On the other hand, measurement of apoB concentration is considerably more expensive and not as available as cholesterol measurements and was not the primary target in intervention trials.

An approximate to apoB is non-HDL-cholesterol, which includes LDL-cholesterol, remnant cholesterol (associated with triglyceride-rich lipoproteins), and lipoprotein (a). Non-HDL-cholesterol can be easily calculated by subtracting HDL-cholesterol from total cholesterol. Non-HDL-cholesterol goals are always $30 \mathrm{mg} / \mathrm{dL}(0.9 \mathrm{mmol} / \mathrm{L})$ higher than LDL-cholesterol goals ${ }^{3}$. Calculating non-HDL-cholesterol and using it as a secondary goal (in addition to LDL-cholesterol) is, therefore, a very appealing option, especially in patients with combined dyslipidemia or diabetic dyslipoproteinemia. Non-HDL-cholesterol is also interesting from a therapeutic point of view, as reaching non-HDL-cholesterol goals can be achieved by either decreasing remnant cholesterol (thus, triglycerides) or by further decreasing LDL-cholesterol.

Concerning hypertriglyceridemia, all guidelines focus on lifestyle changes and glucose control but state that in selected patients, fibrates and/or fish oils can be used to reduce the risk of acute pancreatitis.

In summary, defining LDL-cholesterol and non-HDLcholesterol goals according to the absolute risk and taking into account that LDL-cholesterol should be decreased at least by $50 \%$ is probably the best strategy to prevent cardiovascular disease in high and very high-risk patients.

\section{DO ALL PATIENTS WITH DIABETES REQUIRE STATIN THERAPY?}

This question is very much linked to the previous section. Although it is well known that patients with diabetes mellitus have an increased risk for cardiovascular disease, it should be noted that risk varies widely. For example, some studies indicate that patients with diabetes but no known cardiovascular disease have the same risk as subjects with cardiovascular disease but no diabetes. Other studies indicate that diabetic patients without any additional risk factor have merely a slightly increased risk compared to patients without diabetes 10,11 . Nevertheless, if the goals and recommendations mentioned above are taken seriously, then most adult patients with diabetes should receive statin therapy. Despite these very uniform recommendations, realworld data indicate that even in high-income countries at most a fraction of patients with diabetes is treated with concomitant statin therapy ${ }^{12}$.

\section{LIPID-LOWERING COMBINATION THERAPY IN PATIENTS WITH DIABETES}

This chapter is divided into two parts. The first section refers to the clinical situation when the LDL-cholesterol goal is not achieved with statin therapy; the second section, to the very common situation where the LDL-cholesterol goal is achieved but patients remain more or less hypertriglyceridemic.

When the LDL-cholesterol goal is not achieved with statin therapy (standard dose atorvastatin $20 \mathrm{mg} / \mathrm{d}$ or $40 \mathrm{mg} / \mathrm{d}$ ), combination therapy with ezetimibe $(10 \mathrm{mg} / \mathrm{d})$ should be initiated. This is also supported by an outcome trial showing that diabetic patients had a particular benefit from statin-ezetimibe combination ${ }^{13}$.

It is well known that doubling the statin dose further decreases LDL-cholesterol by approximately $6 \%^{14}$. Therefore, we switch to combination therapy usually if LDL-cholesterol goals cannot be achieved by doses of atorvastatin $40 \mathrm{mg} / \mathrm{d}$ (or equivalent doses of other statins). If this is not sufficient, additional combination with PCSK9-inhibition should be considered. This new class of lipid-lowering drugs can further decrease LDL-cholesterol by $50 \%$ and was also shown to reduce cardiovascular events in patients with and without diabetes ${ }^{15}$. In which diabetic patients PCSK9 inhibitors should be used depends on several factors such as absolute risk (recurrent events) and distance to the goal despite maximally possible lipid-lowering therapy, but also availability and reimbursement situation. A therapy of last resort to further decrease LDL-cholesterol is regular lipoprotein apheresis (if available). An algorithm for achieving the LDL-cholesterol goal is shown in figure 1 . 
Figure 1. Possible algorithm to reach low-density lipoprotein-cholesterol target in patients with diabetes mellitus; local (availability and reimbursement) and individual (contraindication and patient preferences) factors must be considered at all levels before intensifying therapy. (Modified from ${ }^{24}$ )

Define LDL-C target on basis of absolute risk

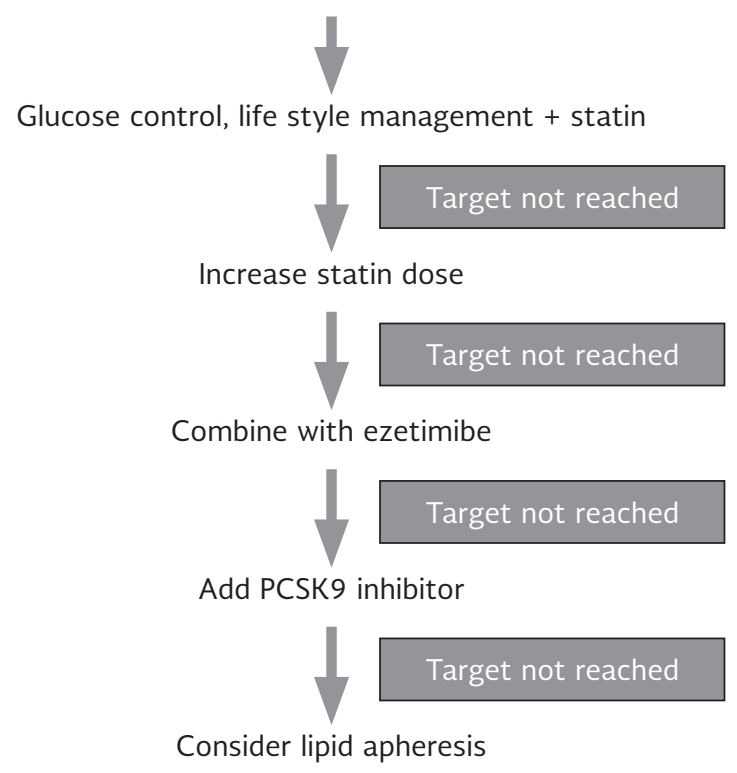

Although the primary goal of lipid therapy in diabetic patients is LDL-cholesterol reduction, the lipid profile of many diabetic patients is characterized by elevated triglycerides ${ }^{16}$. It is known that increased triglycerides mediate an increased cardiovascular risk and may deteriorate glucose metabolism, which raises the question in which patient's triglyceride-lowering drug therapy should be initiated.

Although there is a number of drugs lowering triglyceride concentrations (such as fibrates, niacin/nicotinic acid, and omega-3 fatty acids), their benefit with respect to cardiovascular risk reduction is much less clear than for LDL-cholesterol reduction. This specifically refers to the situation when these drugs are used in combination with statins (Table 3 ). A number of studies (performed 30-40 years ago) showed that fibrate or niacin therapy can result in cardiovascular benefit. Further analyses showed that diabetic patients characterized by the typical diabetic dyslipidemia benefit the most from this approach. Therefore, a specific trial in diabetic patients was designed: FIELD study, which did not show any specific benefit ${ }^{17}$. However, the study was limited by the fact that many people received statin therapy, which presumably diluted the effect of fenofibrate. In another study (AC(ORD), fenofibrate was tested in addition to statins in patients with type 2 diabetes, and no additional benefit was shown ${ }^{18}$. Again severe limitations in the study design should be noted as the patients included in the study were not selected with respect to their lipid profile (i.e., many had normal triglyceride levels). Subgroup analyses and meta-analyses indicate that diabetic patients with typical dyslipidemia (elevated triglycerides, low HDL-cholesterol, and small-dense LDL) may benefit when fibrates are added to statin therapy ${ }^{19}$. This is consistent with a further decrease in non-HDL-cholesterol when fibrates are added to statins. In an ongoing study, fibrates are tested in patients with mixed dyslipidemia. Until the results from this study are available, it remains unclear which patients may benefit from statin-fibrate combinations. This is particularly important as statin-fibrate combinations are characterized by a slightly higher rate of myopathy and even rhabdomyolysis. However, these side-effects are rare if gemfibrozil is omitted, and more modern statins are used.

In our institution, we consider statin-fibrate combinations in diabetic patients at very high risk (usually established atherosclerotic disease) who are at LDLcholesterol goal ( $<70 \mathrm{mg} / \mathrm{dL} ; 1.8 \mathrm{mmol} / \mathrm{L}$ ) on a statin (+ezetibime+PCSK9 inhibition) and still have elevated triglycerides. 
Table 3. Cardiovascular outcome trials with lipid-lowering drugs

\begin{tabular}{lccc}
\hline Drug & $\begin{array}{c}\text { Primary lipid } \\
\text { effect }\end{array}$ & $\begin{array}{c}\text { Outcome trial } \\
\text { monotherapy }\end{array}$ & $\begin{array}{c}\text { Outcome trial in } \\
\text { combination with statins }\end{array}$ \\
\hline Statins & LDL-C $\downarrow$ & + & Not applicable \\
Ezetimibe & LDL-C $\downarrow$ & 0 & + \\
PCSK9 inhibition & LDL-C $\downarrow$ & 0 & + \\
Fibrates & TG $\downarrow, \mathrm{HDL} \uparrow$ & + & - \\
Niacin/nicotinic acid & TG $\downarrow$, LDL $\downarrow, \mathrm{HDL} \uparrow$ & + & - \\
Omega-3-FA & TG $\downarrow$ & + & - \\
\hline
\end{tabular}

+: outcome trial shows cardiovascular risk reduction.

-: outcome trial shows no cardiovascular risk reduction

0 : no outcome trial available.

In summary, it is still unclear which patient groups may benefit from statin-fibrate combinations. Until the results of the ongoing trial are available, this combination should be used in selected high-risk patients. Using omega- 3 fatty acids may be an alternative, but it should be noted that adding omega- 3 fatty acids to statin therapy have not proven to be beneficial ${ }^{20}$. However, again the study design was characterized by several flaws.

Since two outcome trials showed negative results for niacin, this drug should be used very reluctantly ${ }^{21,22}$. In fact, in our institution niacin is not used anymore.

\section{MANAGEMENT OF SEVERE HYPERTRIGLYCERIDEMIA IN PATIENTS WITH DIABETES}

Hypertriglyceridemia is characteristic for patients with diabetes and in some patient's triglyceride values may be $>1000 \mathrm{mg} / \mathrm{dL}(11 \mathrm{mmol} / \mathrm{L})$. Although most of these patients have low LDL-cholesterol levels, they have an increased cardiovascular risk. In addition, the risk of pancreatitis is elevated ${ }^{23}$.

Most outcome trials exclude patients with severe hypertriglyceridemia; thus, it is unclear whether these patients benefit from statin therapy as much as patients with lower triglyceride levels. Nevertheless, statins are rightfully used often in these patients. However, this will usually not decrease triglyceride concentrations sufficiently. Glucose control and reduction of insulin resistance (using adequate antidiabetic drugs) are very important in lowering triglyceride concentrations. Alcohol consumption (even small amounts) and rapidly absorbed carbohydrates (added sugar) and overweight also play important roles. Thus, dietary education is of utmost importance for these patients.

In patients with severe hypertriglyceridemia combination therapy with fibrates and/or omega- 3 fatty acids should also be tested to see whether triglyceride levels can be decreased ${ }^{3,7}$. However, it should be noted that some patients respond very well to such combinations while others do not.

In conclusion, there is no uniform strategy to address severe hypertriglyceridemia in these patients. Glucose control, lifestyle management, weight reduction, adequate antidiabetic medication, and combination therapy of statins with fibrates and/or omega-3 fatty acids are important within this context. In our institution, we usually combine statins with fibrates and omega- 3 fatty acids sequentially to evaluate which combination gives the lowest triglyceride concentrations.

Lipid-lowering therapy is a very potent approach to decrease the cardiovascular risk of diabetic patients. The primary goal should be the reduction of LDLcholesterol using statins or if required, statin-ezetimibe, or statin-ezetimibe-PCSK9 inhibition combinations. Current data indicate that most, if not all adult patients with diabetes should be treated with statins. The indication for triglyceride-lowering drugs is less clear-cut but should be considered in high-risk patients who remain hypertriglyceridemic despite lifestyle modification and adequate glucose control. 


\section{REFERENCES}

1. Collins R, Reith C, Emberson J, et al. Interpretation of the evidence for the efficacy and safety of statin therapy. Lancet. 2016;388:2532-61.

2. American Diabetes Association 9. Cardiovascular disease and risk management: standards of medical care in diabetes-2018. Diabetes Care. 2018;41:S86-S104.

3. Catapano AL, Graham I, De Backer G, et al. 2016 ESC/EAS guidelines for the management of dyslipidaemias. Eur Heart J. 2016;37:2999-3058

4. Parhofer KG, Barrett PH, Schwandt P. Atorvastatin improves postprandial lipoprotein metabolism in normolipidemlic subjects. J Clin Endocrinol Metab. 2000;85:4224-30.

5. Varbo A, Benn M, Tybjærg-Hansen A, et al. Remnant cholesterol as a causal risk factor for ischemic heart disease. J Am Coll Cardiol. 2013:61:427-36.

6. Friedewald WT, Levy RI, Fredrickson DS. Estimation of the concentration of low-density lipoprotein cholesterol in plasma, without use of the preparative ultracentrifuge. Clin Chem. 1972; 18:499-502.

7. Hegele RA, Ginsberg HN, Chapman MJ, et al. The polygenic nature of hypertriglyceridaemia: implications for definition, diagnosis, and management. Lancet Diabetes Endocrinol. 2014;2:655-66.

8. Nordestgaard BG, Langsted A, Mora S, et al. Fasting is not routinely required for determination of a lipid profile: clinical and laboratory implications including flagging at desirable concentration cut-points-a joint consensus statement from the european atherosclerosis society and european federation of clinical chemistry and laboratory medicine. Eur Heart J. 2016;37:1944-58.

9. Sniderman AD, De Graaf J, Couture $P$, et al. Regulation of plasma LDL: the apoB paradigm. Clin Sci (Lond). 2009;118:333-9.

10. Krempf M, Parhofer KG, Steg PG, et al. Cardiovascular event rates in diabetic and nondiabetic individuals with and without established atherothrombosis (from the REduction of atherothrombosis for continued health $[\mathrm{REACH}]$ registry). Am J Cardiol. 2010;105:667-71.

11. Schramm TK, Gislason GH, Vaag A, et al. Mortality and cardiovascular risk associated with different insulin secretagogues compared with metformin in Type 2 diabetes, with or without a previous myocardial infarction: a nationwide study. Eur Heart ]. $2011 \cdot 32: 1900-8$
12. Kotseva K, De Bacquer D, De Backer $G$, et al. Lifestyle and risk factor management in people at high risk of cardiovascular disease. A report from the european society of cardiology european action on secondary and primary prevention by intervention to reduce events (EUROASPIRE) IV cross-sectional survey in 14 european regions. Eur J Prev Cardiol. 2016; 23:2007-18.

13. Cannon CP, Blazing MA, Giugliano RP, et al. Ezetimibe added to statin therapy after acute coronary syndromes. N Engl J Med. 2015;372:2387-97

14. Weng TC, Yang YH, Lin SJ, Tai SH. A systematic review and meta-analysis on the therapeutic equivalence of statins. J Clin Pharm Ther. 2010;35:139-51.

15. Sabatine MS, Giugliano RP, Keech AC, et al. Evolocumab and clinical outcomes in patients with cardiovascular disease. N Engl J Med. 2017;376:1713-22.

16. Parhofer KG. Interaction between glucose and lipid metabolism: more than diabetic dyslipidemia. Diabetes Metab J. 2015 39:353-62.

17. Keech A, Simes RJ, Barter P, et al. Effects of long-term fenofibrate therapy on cardiovascular events in 9795 people with type 2 diabetes mellitus (the FIELD study): randomised controlled trial. Lancet. 2005;366:1849-61.

18. Ginsberg HN, Elam MB, Lovato LC, et al. Effects of combination lipid therapy in Type 2 diabetes mellitus. N Engl J Med. 2010;362:1563-74.

19. Jun M, Foote C, Lv J, et al. Effects of fibrates on cardiovascular outcomes: a systematic review and meta-analysis. Lancet. 2010;375:1875-84.

20. ORIGIN Trial Investigators, Bosch J, Gerstein HC, et al. N-3 fatty acids and cardiovascular outcomes in patients with dysglycemia. N Engl J Med. 2012;367:309-18.

21. AIM-HIGH Investigators, Boden WE, Probstfield JL, et al. Niacin in patients with low HDL cholesterol levels receiving intensive statin therapy. N Engl J Med. 2011;365:2255-67.

22. HPS2-THRIVE Collaborative Group, Landray MJ, Haynes R, et al Effects of extended-release niacin with laropiprant in high-risk patients. N Engl J Med. 2014;371:203-12

23. Pedersen SB, Langsted A, Nordestgaard BG. Nonfasting mild-tomoderate hypertriglyceridemia and risk of acute pancreatitis. JAMA Intern Med. 2016;176:1834-42

24. Parhofer KG. The treatment of disorders of lipid metabolism. Dtsch Arztebl Int. 2016;113:261-8. 